\title{
The COVID-19 Challenge to European Financial Markets Lessons from Italy
}

Nicola Borri

LUISS Guido Carli, Roma, Italia

\begin{abstract}
The COVID-19 pandemic has sickened more than 10 million people around the world and killed at least 500,000. In this chapter, we focus on the experience of Italy, which is the first country hit by the virus in Europe. While the lockdown measures appear to have successfully contained the virus, the economic consequences have been very severe. Policy makers should study the Italian experience to evaluate the cost-benefit effectiveness of different policies in containing the pandemic.
\end{abstract}

Keywords COVID-19. Italy. Pandemic. Lockdown. ECB.

Summary 1 Introduction. -2 The Evolution of the Health Crisis in Italy. -3 The Government Response to the Pandemic. 4 The Economic Effects of the Pandemic. 5 Conclusions.

\section{Introduction}

As a result of the COVID-19 pandemic, nearly 3 billion people worldwide have been under lockdowns put in place by governments to stop the virus, with severe consequences for workers, firms, and public finances. The pandemic is also expected to have long-run effects on the economy and individuals' wellbeing which cannot be fully assessed yet.

The diffusion of the pandemic, according to data and evidence currently available, was very fast. The starting point is usually traced back to Wuhan (China), at the end of 2019, when dozens of cases from an unknown virus were registered. In January 2020, Japan and South Korea confirmed the 
first cases outside mainland China, and towards the end of February 2020 infections surged in South Korea and Italy. Since then, most countries in the world have been affected, although with different intensities, in part depending on the type and timing of social distancing measures in place.

In this chapter, we focus on the experience of Italy, which is the first country hit by the virus in Europe. We believe the example of Italy is of particular interest for at least three reasons. First, Italy is considered approximately 2-3 weeks ahead of other advanced economies in the state of the pandemic. Second, Italy put in place one of the stricter lockdown policies, practically halting a large fraction of the economic activities and the movement of people not only across the country, but also within cities and towns. Therefore, Italy is an interesting case study to evaluate the effectiveness of different policies in fighting the pandemic and their economic and social costs. Third, because Italy is one of the advanced countries with the most fragile government finances, and with a stagnant economy for at least a decade, the COVID-19 shock could spark an economic crisis which could potentially propagate to the Eurozone and beyond.

The rest of the chapter is organised as follows. Section 2 describes the evolution of the health crisis in Italy. Section 3 presents the government response to the health emergency. Section 4 describes the economic effects of the pandemic in Italy. Finally, in Section 5 we present our conclusions.

\section{The Evolution of the Health Crisis in Italy}

After four months since the outbreak of the pandemic, the situation in Italy is finally stabilising, but the death toll is dramatic. The number of active cases is declining, as is the number of daily deaths due to COVID-19, which is approaching zero. Figure 1, using official data from the Italian Protezione Civile, shows a breakdown of the evolution of the pandemic in Italy into active cases (roughly 16 thousand at the end of June), deaths (roughly 35 thousand at the end of June) and recovered cases (roughly 200 thousand at the end of June) [fig. 1]. The figure shows that the number of active cases reached a peak at the end of April 2020, and then slowly declined as the number of recovered cases increased. Differently from other countries, like the US, where the diffusion of the pandemic resumed to grow after a first stabilisation, current data for Italy do not show any resurgence of the virus.

Figure 2 considers alternative measures of the state of the pandemic, and in particular the number of patients hospitalized or in quarantine [fig. 2].

One of the lessons from the Italian experience with the pandemic is that congestion of hospitals, and in particular of intensive care units 
Nicola Borri

The COVID-19 Challenge to European Financial Markets. Lessons from Italy

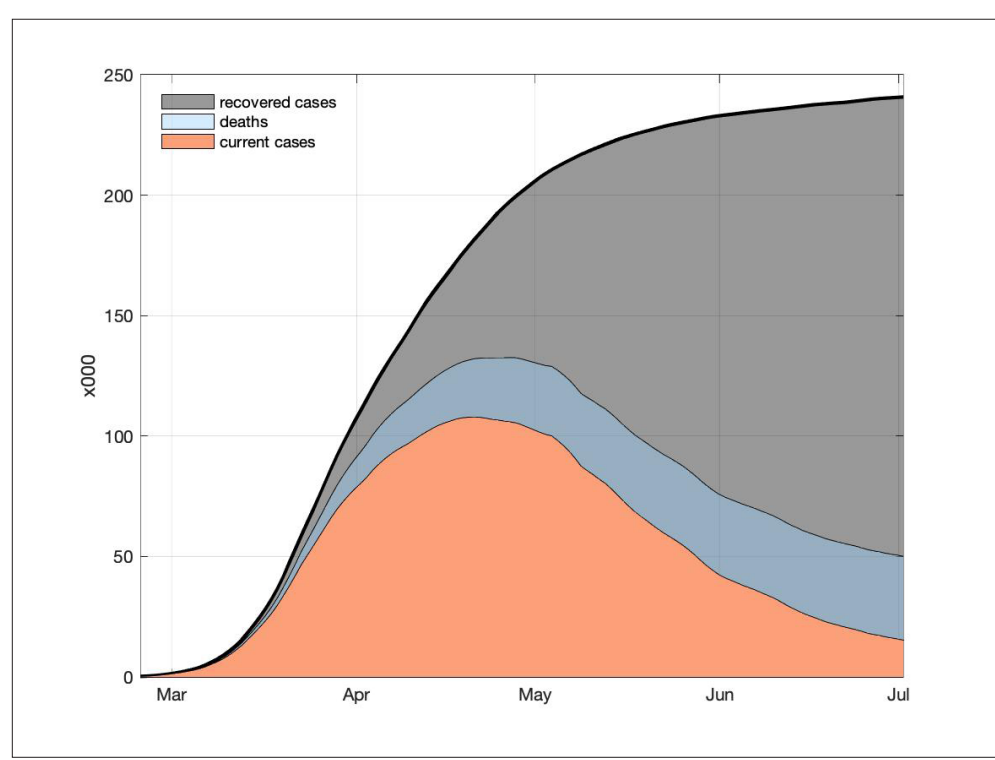

Figure 1 The Evolution of the COVID-19 Pandemic in Italy. Notes: the figure shows the evolution of the COVID-19 pandemic in Italy. The black solid line corresponds to the cumulated number of cases; the orangeshaded are active cases; the blue-shaded area are COVID-19 deaths; the gray-shaded area indicates recovered

cases. Data are from Protezione Civile and available at: https://github.com/pcm-dpc/COVID-19

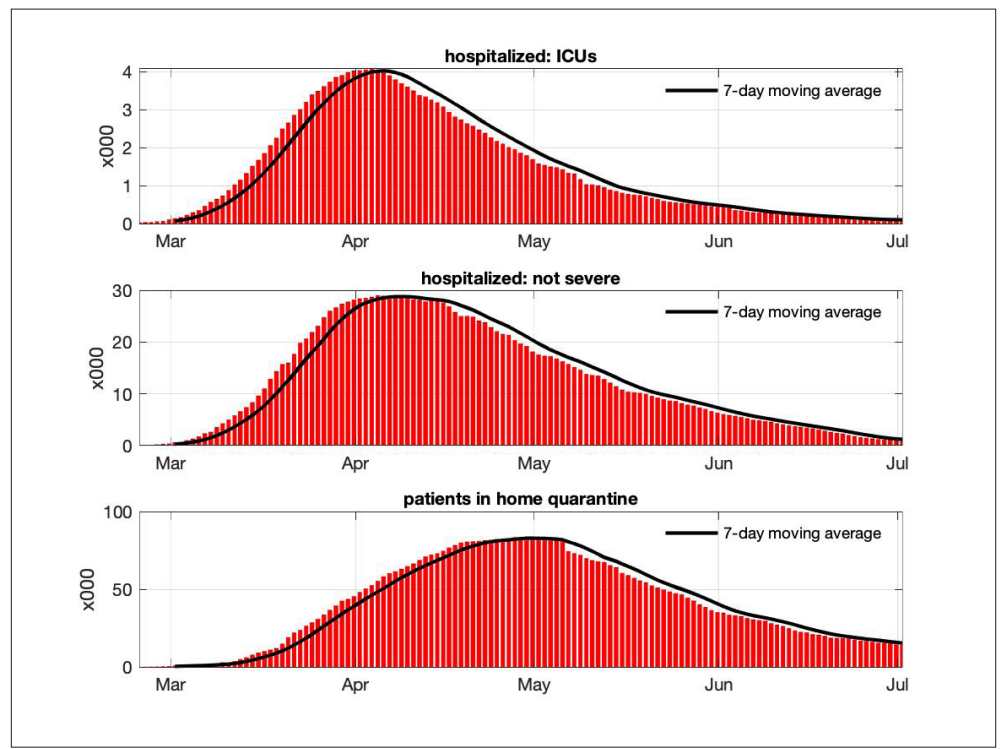

Figure 2 Patients Hospitalized and in Quarantine. Notes: the figure plots the evolution of the patients hospitalized in ICUs (top panel); hospitalized, but in less severe conditions (middle panel); and in quarantine at home (bottom panel). The black solid lines correspond to the 7-day moving average. Data are from Protezione Civile and available at: https://github.com/pcm-dpc/COVID-19 
(ICUs), should be avoided at all costs. In fact, given the exponential path of the uncontrolled pandemic, hospitals are likely to work at capacity, unable to cure all COVID-19 patients, and forced to postpone non-essential treatment to non COVID-19 patients with health consequences that are difficult to predict. The current data show that both the number of patients in ICUs, and the number of hospitalized patients in less severe conditions, are currently steadily declining (see the top and middle panels of figure 2). Specifically, the number of patients in ICUs reached a maximum value of approximately 4,000 in April, at the peak of the pandemic. As the diffusion of the pandemic was particularly severe in the North of the country, ICUs in these regions worked at full capacity, and this might have increased the true death toll (e.g. Bonanno, Galletta, Puca 2020; Galeotti, Surico 2020). Finally, the bottom panel of figure 2 shows the evolution of the patients in quarantine at home which has also been steadily declining. Although these are patients with less severe symptoms, they risk infecting their relatives and/or anyone living in their homes.

The diffusion of the pandemic in Italy has been very heterogenous across the Italian regions. Specifically, the pandemic was concentrated in the North of the country, and in particular in Lombardia, Piemonte, Veneto and Emilia Romagna. These regions account for approximately $40 \%$ of the Italian gross domestic product (GDP) and are populated by a large number of firms with interactions with firms scattered around the world. This could have been at the origin of the first contagion with the COVID-19 virus. Figure 3 shows the number of current active cases, along the number of cases at the beginning of three different phases of the lockdown (described in the next section), at the province level (note that these numbers are not divided by population) [fig. 3]. A quick look at the figure immediately reveals the strong geographic component of the pandemic and indicates that the lockdown was successful in containing the spreading of the virus throughout the country.

It is important to notice that the heterogeneity in the diffusion of the pandemic does not depend on, for example, differences in the frequency of testing. For example, we observe similar heterogeneity in the evolution of the COVID-19 deaths across Italian regions. Figure 4 shows that approximately $65 \%$ of the total COVID-19 deaths occurred in five regions: Lombardia, Piemonte, Emilia Romagna, Veneto and Liguria [fig. 4]. 
Nicola Borri

The COVID-19 Challenge to European Financial Markets. Lessons from Italy

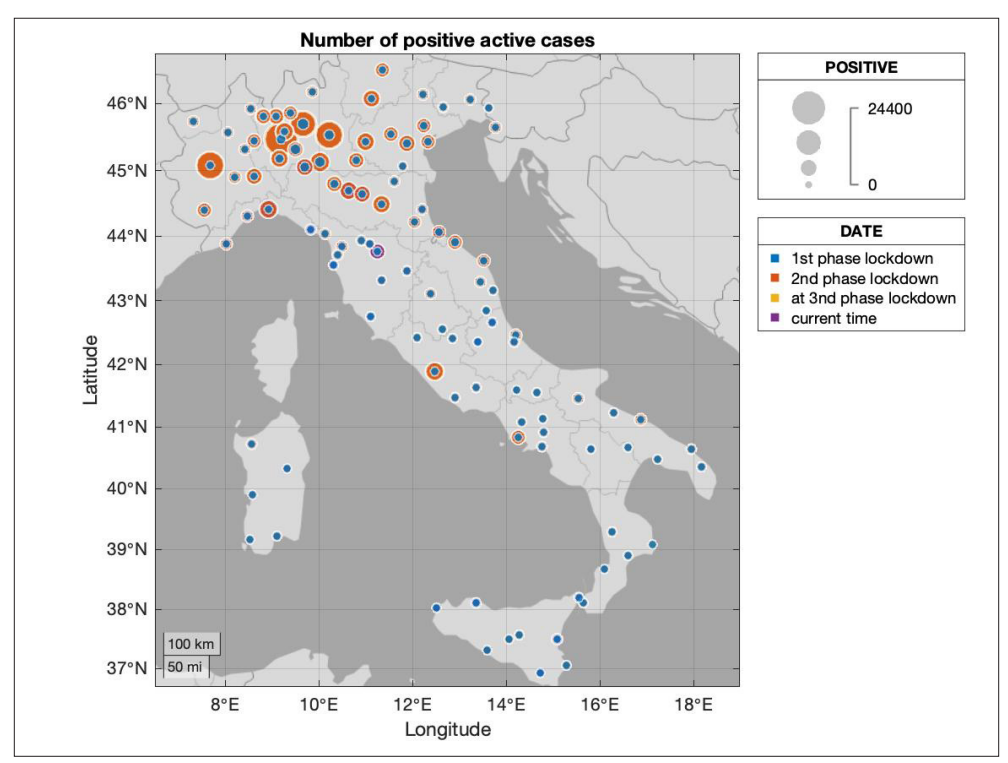

Figure 3 Number of COVID-19 Positives. Notes: The figure plots the total number of active COVID-19 cases at the province level. The size of the circles increases with the number of active cases. The colors correspond to different dates: first phase of national lockdown (blue, March 9, 2020); second phase of national lockdown (orange, May 4, 2020); third phase of national lockdown (yellow, June 15, 2020); current time (violet, June 30 , 2020). Data are from Protezione Civile and available at: https://github.com/pcm-dpc/COVID-19

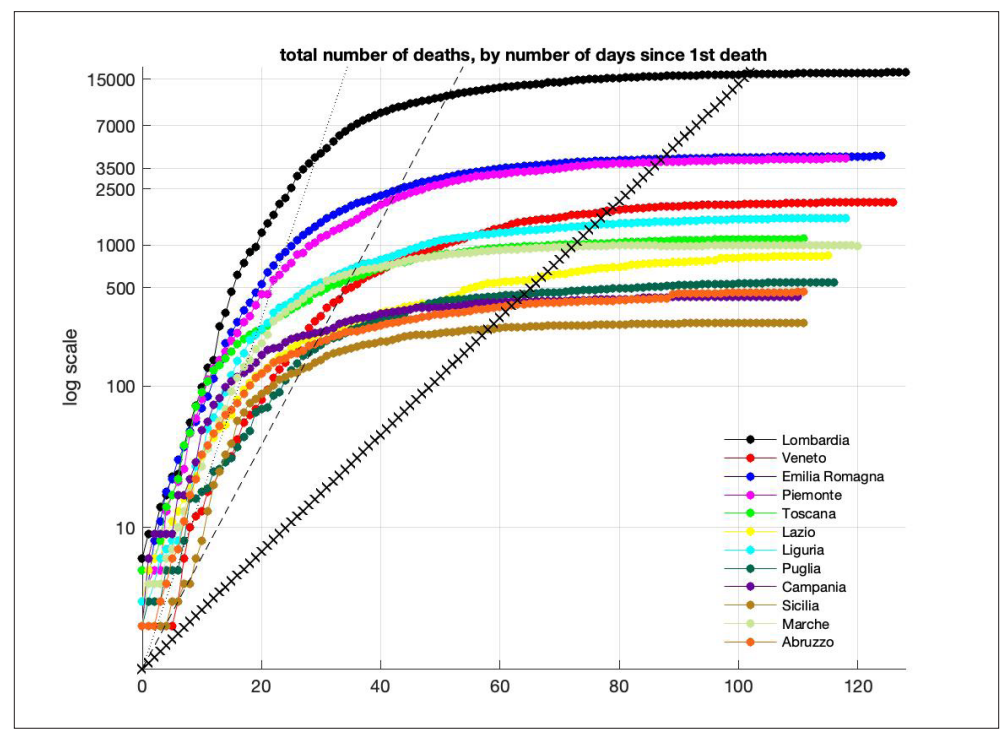

Figure 4 Evolution COVID-19 Deaths at Regional Level. Notes: The figure plots the evolution of the COVID-19 deaths for a sample of Italian regions. For each region, the values on the horizontal axis correspond to the number of days since the first COVID-19 death. The $y$-axis is in log-scale, the $y$-ticks correspond to numbers in levels. The straight lines from the origin correspond to daily growth rates of, respectively, $10 \%, 20 \%$, and 33\%. Data are from Protezione Civile and available at: https://github.com/pcm-dpc/COVID-19 


\section{The Government Response to the Pandemic}

In this section we consider the response of the Italian government to the health emergency, while in the next section we additionally consider the government response to the economic crisis.

The response of the Italian government to the pandemic has been a strict lockdown. We can roughly distinguish three phases of the lockdown. The first phase started on March 9 in all regions and implied the closing of schools, some economic activities (for example, restaurants, hospitality and non-food retail sectors), and the restriction of movement of people not only across regions, but also within regions and municipalities. In addition, on March 23 all non-essential economic activities were also shut down, affecting approximately $51 \%$ of firms and $55 \%$ of workers. The lockdown of economic activities, because of the heterogeneity in the geographical distribution of essential activities, determined differences in the fraction of inactive workers across different provinces [fig. 5].

Borri, Drago, Sobbrio (2020) used exactly this policy induced heterogeneity in the fraction of inactive workers to evaluate the effectiveness of the economic lockdown. Table 1 summarises their results. Specifically, while the estimated effect of the share of inactive workers goes in the right direction and is associated to a reduction in the number of COVID-19 infected patients, the estimates are not statistically significant. Borri, Drago, Sobbrio (2020) argue that their results, rather than evidence against the effectiveness of the lockdown, highlight the importance of good quality granular data, for example at the municipal level, and/or accounting for mobility patterns.

Table 1 Effect of the economic Lockdown

\begin{tabular}{lccc}
\hline & \multicolumn{2}{c}{ COVID-19 positive cases (province level) } \\
\hline & All provinces & $\begin{array}{c}\text { Provinces } \\
\text { in North }\end{array}$ & $\begin{array}{c}\text { Provinces above median } \\
\text { at lockdown }\end{array}$ \\
\hline$\Delta$ inactive post-lockdown & -139.977 & -356.936 & -305.268 \\
\hline & $(87.030)$ & $(218.068)$ & $(229.712)$ \\
\hline Obs. & 3640 & 1540 & 1540 \\
\hline FE province level & YES & YES & YES \\
\hline FE region-day & YES & YES & YES \\
\hline $\begin{array}{l}\text { Unconditional mean } \\
\text { dependent variable }\end{array}$ & 796.8 & 1496 & 1556 \\
\hline
\end{tabular}

Notes: robust standard errors clustered at provincial level. Regression includes five lags of dependent variable. The table report the estimates of a panel estimation model for the COVID-19 positive cases (at the province level) on the share of inactive workers as a consequence of the lockdown. The estimations always include a fixed effect at the province level; a fixed effect at the region-day level. The table is from Borri et al. 2020. 


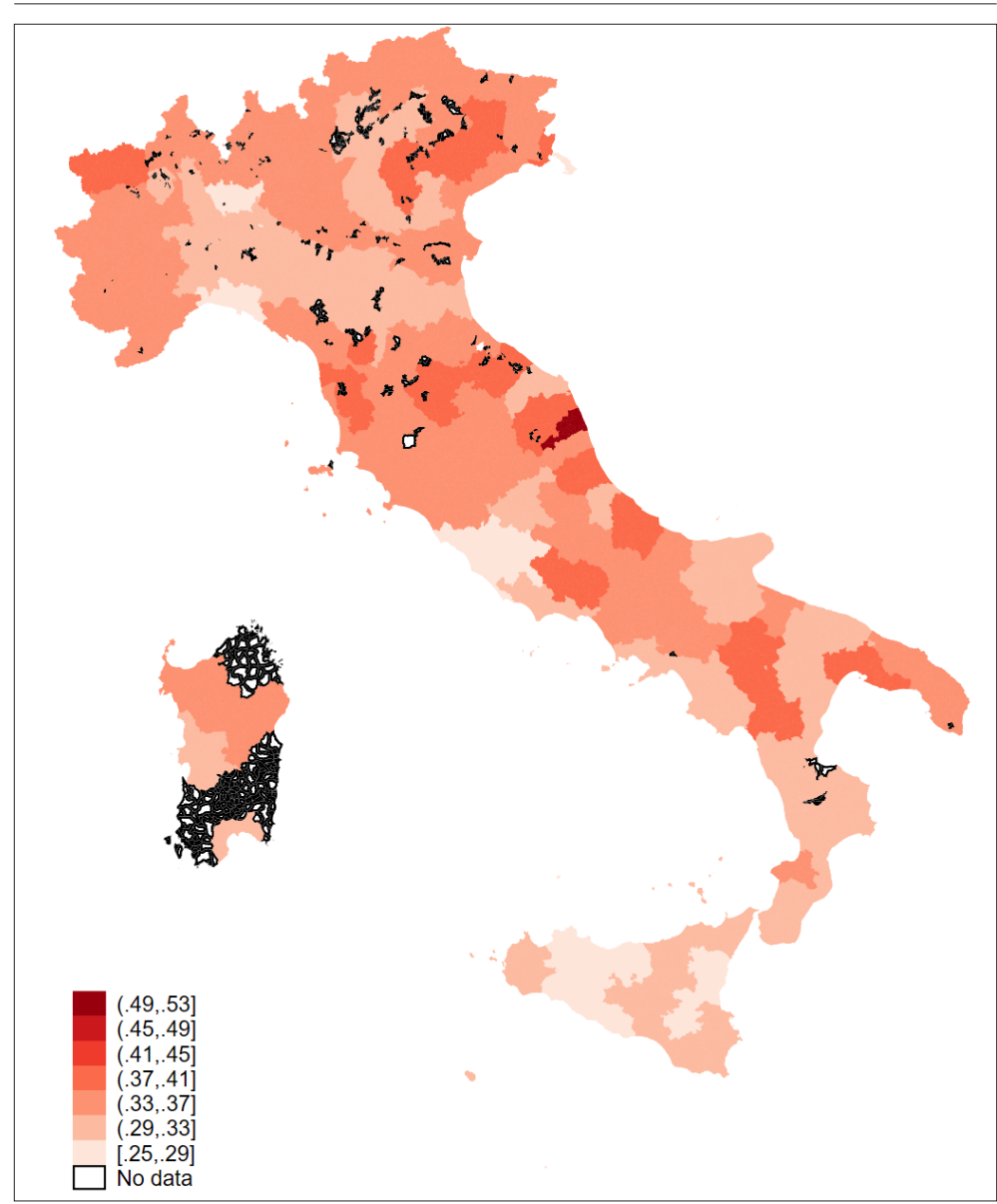

Figure 5 Share of Inactive Workers. Notes: The figure represents the share of inactive workers as a consequence of the first phase of the economic lockdown. Dark (lighter) colours correspond to a higher (lower) fraction of inactive workers. The figure is from Borri et al. 2020

The second and third phases correspond to the relaxation of the lockdown. Specifically, the second phase started on May 4, with the reopening of economic activities, and the relaxation of the restrictions to the movement within regions. Finally, the third phase started on June 15, and implied the relaxation of most lockdown measures. However, all schools remained closed and the use of masks in public places is currently mandatory. It is too early to be able to evaluate the risks and/or effects associated to the relaxation of the lockdown measures in the second and third phases in Italy. 


\section{The Economic Effects of the Pandemic}

While the lockdown, decided by the Italian government, has been so far effective at containing the pandemic, its economic effects have been very severe. Italian GDP is expected to drop by approximately 10\% in 2020 (Bloomberg). This historically large drop in GDP depends on the expected decrease in consumption (-11.8\%), investment (-15.4\%), and industrial production (-14.5\%). Government deficit is expected to be large and equal to $12.2 \%$ and, correspondingly, public debt is expected to reach $150 \%$ of GDP. The large increase in public debt depends on both the increase in public spending put in place to limit the negative effects of the pandemic (i.e. unemployment benefits, credit guarantees to firms, healthcare investments, etc.), and the sharp reduction in tax revenues because of the lower output. The fiscal response by the Italian government has been so far limited and constrained by the large public debt. The approved measures, worth around 1\% of GDP, are associated to emergency financing of the health system; employment and income support; tax deferrals and utility bills; and support of credit supply. Most analysts also expect a quick recovery in 2021, after the end of the lockdown. For example, for 2021, they expect an increase in GDP of approximately 5.6\%. However, there is substantial uncertainty around all these estimates, as well as over the likelihood that a second COVID-19 wave could hit advanced economies in the Fall/Winter of 2020.

Because of the large public debt and low economic growth before the COVID-19 shock, Italy is particularly exposed to the recent crisis. In addition, the fragile public finances limit the resources that the government could inject in the economy, thus increasing the risk of a deep recession. In fact, Italy is expected to be one of the economies most severely hit in the Eurozone. The response of equity markets, which are forward looking, confirms the difficult economic Italian outlook. Figure 6 shows the evolution of three equity indices, normalised to 1 on January 1, 2020 [fig. 6]. The equity indices correspond to the S\&P500 for US equity markets, the Eurostoxx50 for Eurozone equity markets, and the FTSE MIB for the Italian equity market (data are from Bloomberg). The figure shows that while the initial drop in the equity market in Italy and the rest of the Eurozone was similar, the recovery has been weaker in Italy. In fact, equity markets dropped, first, by roughly $35 \%$ in Italy and the rest of the Eurozone, and by $30 \%$ in the US. The intervention by the major central banks, which announced large asset purchase programmes like the Pandemic Emergency Purchase Program (PEPP) by the European Central Bank (ECB), helped to put a floor to the fall in equity markets. However, while the US equity market has gained back almost all of the lost ground, the Eurozone market and, especially, the Italian market, are still approximately $15 \%$ lower than their values at the beginning of the year. 


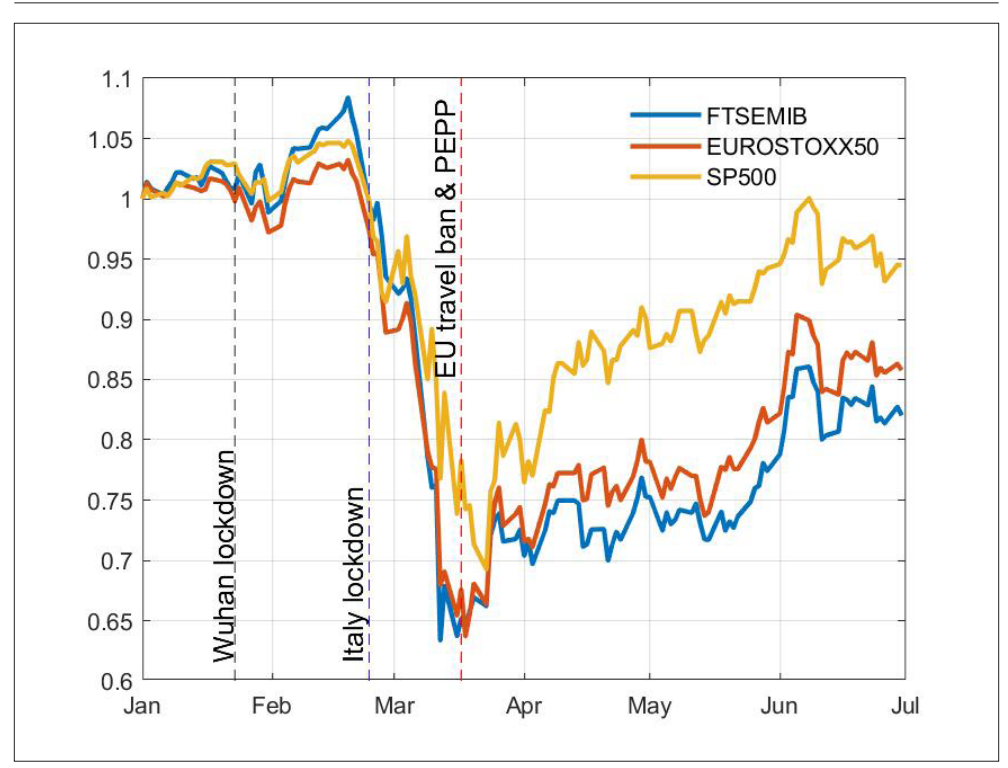

Figure 6 Equity Markets around the Great Lockdown. Notes: the figure shows the evolution of the prices of the S\&P500 (solid yellow line), Eurostoxx50 (solid red line), and FTSE MIB (solid blue line) indices. All series are normalised to 1 on January 1, 2020. The vertical lines correspond to the Wuhan lockdown, the first lockdown in Italy limited to some regions in the North, and the announcement of the ECB PEPP programme. Data are from Bloomberg

Because investors and policy makers expect a large increase in public debt, and a significant fall in output and tax revenues, they also worry about the possible default of one, or more, members of the Eurozone, overwhelmed by a striking crisis and thus compelled to stop servicing their debt. Figure 7 shows the evolution of government bond yields for Italy, Spain and Portugal (the data are from Bloomberg) [fig. 7]. As we have seen for equity markets, also for government bonds we first see a sharp increase in yields (and, thus, a sharp drop in prices). For example, the yield on the 10-year Italian government bond increased from 150 to more than 250 basis points. We observe a similar increase in yields also for Spain and Portugal which, however, had lower yields before the COVID-19 shock. Even so, it appears that the intervention by the ECB, with the announcement of the PEPP asset purchase programme, was able to avoid the risk of a sovereign debt crisis.

Bonaccolto, Borri, Consiglio (2020) find also evidence of an increase in the redenomination risk for France and Italy since the beginning of January 2020, i.e. the risk that one, or both, of these two countries abandon the Euro for a new, undervalued, currency. However, they also find that this risk is historically lower than after political shocks, like the recent election in Italy of an anti-euro government. 


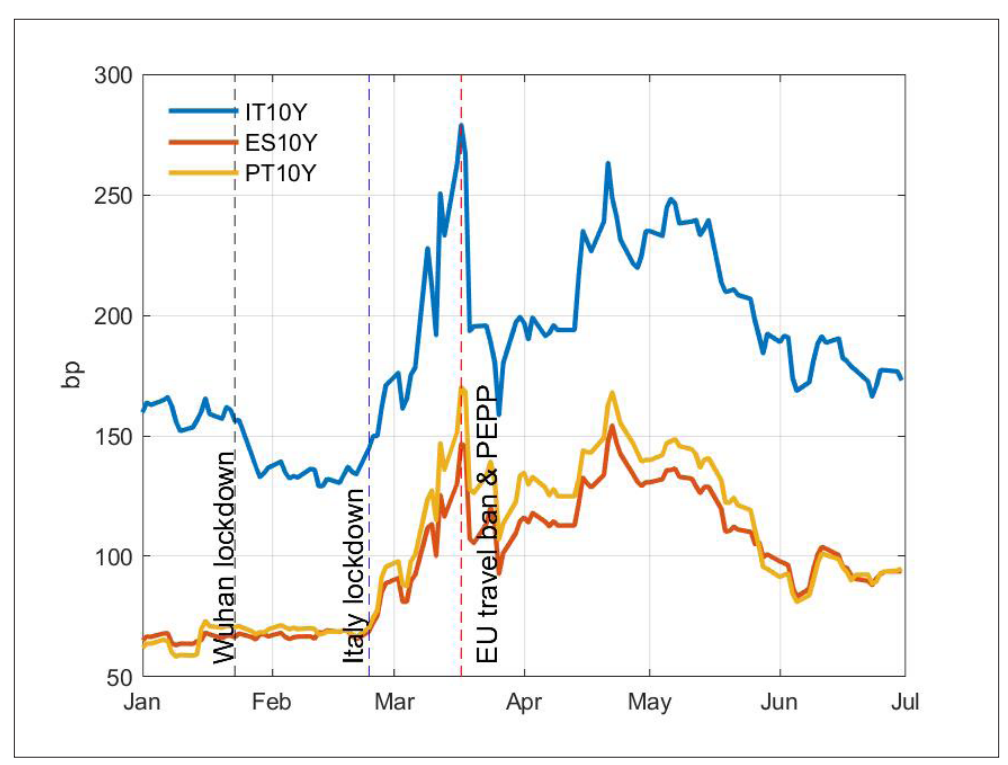

Figure 7 Government Bond Yields around the Great Lockdown. Notes: the figure shows the evolution of the yields of the Italian (solid blue line), Spanish (solid red line), and Portuguese (solid yellow line) 10-year benchmark government bonds. The vertical lines correspond to the Wuhan lockdown, the first lockdown in Italy limited to some regions in the North, and the announcement of the ECB PEPP programme. Yields are reported in basis points. Data are from Bloomberg

\section{Conclusions}

Italy is one of the first advanced economies hit by the COVID-19 pandemic. After approximately four months, the health crisis has stabilised, and the pandemic appears to be under control. However, there is still a lot of uncertainty around the possibility that a second wave could hit the country in the next Fall.

One of the lessons from the Italian experience is that strict lockdown measures are necessary to stop the pandemic and avoid that the virus spreads across regions. In addition, large scale testing is crucial to identify positive cases with no or small symptoms. In fact, within Italy, we observe very different outcomes for two neighbouring regions, Lombardia and Veneto. Although these two regions were the first to be hit by the COVID-19, they subsequently experienced very different evolutions of the pandemic, with Lombardia suffering many more deaths. While the Veneto region started early with a large testing programme which was effective at identifying positive cases, the Lombardia region started a large testing programme only at a later date. Finally, the strict restriction to the movement of people within the country is likely to be one of the reasons for the virus not to spread to, for example, the South of the country. 
While the lockdown measures appear to have successfully contained the virus, the economic consequences have been very severe. The economic situation is very fragile, and critically supported by international institutions, like the ECB, which has been able to avoid the risk of a sovereign debt crisis. This experience confirms the need for a risk-sharing mechanism in the Eurozone. So far, the actions of the ECB have filled this hole in the design of the European institutions. However, for the future, it is crucial to think of new, and permanent, instruments that guarantee some forms of risk-sharing.

In this light, an objective cost-benefit analysis of lockdown measures is crucial for at least three reasons. First, to guide and inform policy makers in their decisions regarding a safe and effective relaxation of the lockdown measures after the end of the medical emergency. Second, to guide and inform policy makers in the design of the social distancing measures that are likely to be in place up until a vaccine against COVID-19 has been found or that should immediately become effective in the event of a second 'wave' of the pandemic. Third, to understand the overall cost-effectiveness of the different lockdown measures in the containment and/or prevention of future pandemics. In fact, because different "nonpharmaceutical interventions" (NPIs) differ from each other in terms of their economic and psychological cost, it is crucial "to identify the interventions that most reduce transmission at the lowest economic and psychological cost" (Haushofer, Metcalf 2020).

This type of analysis remains, however, a daunting task, as it hinges upon the evaluation of a causal effect of these measures on the spread of the pandemic, or the economy (see, for example, GoodmanBacon, Marcus, 2020). While challenging, the heterogeneity in the introduction of these measures in Italy - both at the geographical and the temporal level - and the availability of detailed data on mobility and deaths at the city level provide researchers with a unique quasi-experimental pre-test/post-test design to elicit the causal effects of the lockdown. 


\section{Bibliography}

Bonaccolto, G.; Borri, N.; Consiglio, A. (2020). "Breakup and Default Risks in the Great Lockdown”. Working Paper. https://dx.doi.org/10.2139/ ssrn.3487453.

Bonanno, P.; Galletta, S.; Puca, M. (2020). "Estimating the Severity of Covid-19: Evidence from the Italian Epicenter". Working Paper. https://dx.doi. org/10.2139/ssrn.3567093.

Borri, N.; Drago, F.; Sobbrio, F. (2020). “Lockdown dell'economia, un primo bilancio". Lavoce, 20 April. https://www.lavoce.info/archives/65771/ lockdown-delleconomia-un-primo-bilancio/.

Galeotti, A.; Surico, P. (2020). "A User Guide to COVID-19”. VoxEU. https:// voxeu.org/article/user-guide-covid-19.

Goodman-Bacon, A.; Marcus, J. (2020). "Using Difference-in-Difference to Identify Causal Effects of COVID-19 Policies”. Working Paper. https://doi. org/10.18148/srm/2020.v14i2.7723.

Haushofer, J.; Metcalf, J. (2020). "Which Interventions Work Best in a Pandemic?”. Science, 368(6495), 1063-5. http://doi.org/10.1126/science. abb6144. 